\title{
Outcome Evaluation of HER2 Breast Cancer Patients with Limited Brain Metastasis
}

\author{
FEDERICO PESSINA $^{1}$, PIERINA NAVARRIA ${ }^{2}$, LUCA COZZI $^{2,3}$, DAVIDE FRANCESCHINI ${ }^{2}$, \\ STEFANO TOMATIS ${ }^{2}$, ELENA CLERICI ${ }^{2}$, ANNA MARIA ASCOLESE ${ }^{2}$, FIORENZA DE ROSE $^{2}$, \\ LORENZO BELLO ${ }^{1}$, GIOVANNA MASCI $^{4}$, ARMANDO SANTORO ${ }^{3,4}$ and MARTA SCORSETTI ${ }^{2,3}$ \\ ${ }^{1}$ Neurosurgical Oncology, Humanitas Cancer Center and Research Hospital, Rozzano-Milan, Italy; \\ ${ }^{2}$ Radiotherapy and Radiosurgery, Humanitas Cancer Center and Research Hospital, Rozzano-Milan, Italy; \\ ${ }^{3}$ Department of Biomedical Sciences, Humanitas University, Rozzano-Milan, Italy; \\ ${ }^{4}$ Haematology and Oncology, Humanitas Cancer Center and Research Hospital, Rozzano-Milan, Italy
}

\begin{abstract}
Background/Aim: To appraise the outcome of limited brain metastasis (BMs) from HER2 breast cancer. Patients and Methods: The study cohort included sixty-six patients Treatments consisted of surgery followed by radiotherapy (RT), or radiosurgery (SRS/HSRS) or whole brain radiotherapy (WBRT). Results: Surgery followed by RT was performed in $25.8 \%$ of patients, SRS/HSRS alone in $48.5 \%$, and WBRT alone in $25.7 \%$. The median follow-up time was 23.4 months and 32.5 months for alive patients. Local recurrence occurred in $16(24.2 \%)$ patients. The median, 1-, 2-, 3-year local control were (median not reached $=n r), 87.5 \%, 71.2 \%$, and $63.0 \%$. The median $1-, 2-$, 3-year overall survival were 30.7 months (95\% CI: 18.9-42.6 months), $78.5 \%, 57.4 \%, 43.3 \%$. Karnofsky Performance Scale (KPS), number of BMs, local treatment performed, the presence of extracranial (EC) metastases at the time of BMs diagnosis, and the administration of trastuzumab affected survival. Conclusion: HER2BC patients with good performance status, controlled EC disease and single BM had better outcome. In this long-term survivor patients, local BMs treatment can affect survival.
\end{abstract}

Breast cancer (BC) is the second most common cause of metastatic brain metastases (BMs), and although they are rarely found at diagnosis of primary tumor, the risk of developing them has been reported in the range of $10-15 \%$ of cases during the course of disease $(1,2)$. The advances in neuroimaging and

Correspondence to: Dr. Luca Cozzi, Humanitas Cancer Center and Research Hospital, Via Manzoni 5620089 Rozzano-Milan, Italy. Tel: +41 796176321, Fax: +39 0282248509, e-mail: luca.cozzi@ humanitas.it

Key Words: Brain metastases, radiosurgery, surgery, targeted therapy. the availability of biomarker based therapy has led to an increased BMs diagnoses, to the extent of $20-25 \%$, according to the BC tumor phenotype. The greater incidence was recorded in cases of triple negative (TN) or HER2BC subtypes (3-9). TNBC have a high propensity to develop CNS disease, already at the time of primary tumor diagnoses. The outcome is particularly poor, and patients succumb quickly for progressive extracranial (EC) disease, early BMs recurrence following radiation therapy, or both, with a median survival time of about 6 months $(10,11)$. On the other hand, in HER2BC patients BMs, more commonly, arise later, while the patient is receiving HER2-targeted therapy for EC metastases, or whilst EC disease is well controlled, or even when its progression may be controlled with another line of HER2targeted therapy. The median survival time from occurrence of BMs is between 18 and 23 months in relation to the HR status. Regarding this group of patients, although HER2-directed antibodies are able to control EC disease, unfortunately they poorly penetrate the blood brain barrier, and appear to provide an unclear benefit for patients with BMs $(12,13)$. In addition, retrospective series suggest that neurological death, for uncontrolled metastatic intracranial disease rather than from systemic disease, may be more common in HER2BC patients than for the other phenotypes (14). Therefore, in this set to identify the most effective local treatment would seem advisable. Local treatments of BMs include surgical resection, whole brain radiation therapy (WBRT), single dose radiosurgery (SRS) or hypofractionated radiosurgery (HSRS). In case of large BMs, surgical resection combined with various RT modalities obtained a 1-year local control (LC) rate $>80 \%$, significantly influencing survival $(15,16)$. Adjuvant WBRT has proven to reduce the risk of local recurrence from $46-59 \%$ to $10-28 \%$ and the incidence of new BMs from $37-42 \%$ to $14-$ $23 \%(17,18)$. Nevertheless, considering the potentially risk of neurocognitive impairment without an improvement in survival, localized adjuvant treatments on the tumor bed, such 
as SRS or HSRS, have been investigated and increasingly used in place of WBRT (19-22). For small brain lesions SRS represents the preferred local treatment, achieving a 1-year LC rate of $90 \%(23,24)$. Emerging data suggest that the targeted therapies, able to control extracranial disease, combined with local treatments, are the way forward to improve outcome (2528). Based on this observation, we retrospectively evaluated HER2BC patients with limited BMs (up to 4) treated in our institution. The primary aim of this analysis was to assess the impact of different kinds of treatments performed (surgery, WBRT, SRS or HSRS) on local control (LC) and overall survival (OS) and the eventual advantage of the targeted therapy administration.

\section{Patients and Methods}

The present retrospective study includes patients with limited brain metastases $(\leq 4)$ from primary HER2BC treated in our institution. All patients received at the time of primary diagnosis neo-adjuvant or adjuvant chemotherapy followed by HER2 antibodies (trastuzumab). The most frequent chemotherapeutic agents used were adriamicina, ciclophosfamide and docetaxel. In case of HER2HR positive, hormone therapy was administered too. Local treatments consisted of combined approaches, surgery plus HSRS or surgery plus WBRT, SRS or HSRS alone, and WBRT only. Surgical resection was performed in case of patients with KPS $\geq 70$, controlled extra-cranial disease, single large brain lesion or presence of multiple BMs of which one conditioning mass effect and progressive neurological deficits, refractory to medical therapy. SRS alone in case of small and multiple BMs; HSRS or WBRT alone in case of large BMs in patients with KPS $<70$, and not controlled extra-cranial disease. To define the appropriate therapy, each patient was evaluated by a multidisciplinary team including oncologists, radiation oncologists and neurosurgeons. A "supramarginal resection", defined as a microsurgical tumor excision with an extension at least $5 \mathrm{~mm}$ larger than enhancing $\mathrm{T} 1$ weighted MRI sequences borders, was performed. In case of dural attachment the dura mater removal was carried out at least $2 \mathrm{~cm}$ from the stalk in each direction. In order to precisely delineate the target volume for SRS or HSRS plans, enhanced T1-MRI sequences and post-contrast CT scans were used and co-registered. The clinical target volume (CTV) corresponded to the lesion or the tumor bed; a planning target volume (PTV) was generated adding an isotropic expansion of $2 \mathrm{~mm}$ from CTV. All plans were optimized on PTV. The prescribed total doses were $24 \mathrm{~Gy} / 1$ fraction for lesions $\leq 3 \mathrm{~cm}$ in maximum diameter and $30 \mathrm{~Gy}$ (range $=24-40 \mathrm{~Gy}$ ) in 3-10 fractions for lesions $>3 \mathrm{~cm}$ or located in close proximity to visual apparatus (optic chiasm or optic nerves). After local treatments, trastuzumab and/or hormonal therapy were used.

Outcome evaluation. Clinical outcome was evaluated by brain MRI performed two months after RT and then every 3 months. Local progression was defined as radiographic increase of the enhancing abnormality in the irradiated volume, and brain distant failure (BDF) as the presence of new BMs outside the irradiated volume. Systemic disease was evaluated by post-contrast total body CT-scan, 18-FDGCT-PET and CA 15.3 blood quantification. Prognostic factors analyzed were age, KPS, stage at diagnoses, primary tumor subtype, interval time between primary tumor diagnoses and appearance of BM, RPA class, breast specific GPA score (8), and presence of other metastatic sites at time of BMs, number.

Statistical analysis. Standard descriptive statistics (mean, standard deviation and cross tabulation analysis) were used to describe the general data behavior. Survival and recurrence time observations were plotted according to the Kaplan-Meier method, starting from the date of BMs diagnosis. The log-rank test was used to carry out the univariate analysis, in order to investigate the prognostic role of individual variables. Groups were defined according to discrete volume of each variables. Multivariate Cox model was used as a method to estimate the independent association of a variable set with overall survival, local control, and brain distant failure. Statistical software used was SPSS v.22 (IBM Corp, Armonk, USA).

\section{Results}

From June 2005 to November 2015, 66 patients with limited BMs from primary HER2BC, were treated. The median age was 52 years (range $=31-83$ years). At the time of primary HER2BC diagnosis $16(24.2 \%)$ patients were in stage IV and $50(75.8 \%)$ in stage I-III; regarding tumor subtype $27(40.9 \%)$ had HR-positive status and 39 (59.1\%) negative status. For the entire cohort, the median interval time between the diagnosis of primary HER2BC and the appearance of BMs was 39.8 months (range=3.8-189.1 months), 41.8 months (range=8.4-146.4 months) for HER2HR positive, and 38 months (range=3.8-189.1 months) for HER2HR negative. At the time of BMs, $48(72.7 \%)$ patients had other extra-cranial metastases and $18(27.3 \%)$ BMs only. The majority of patients had a KPS 90-100 (65.0\%), were in RPA class II $(72.7 \%)$, and had $1-2 \mathrm{BMs}(71.2 \%)$. Details about patients and tumor characteristics are shown in Table I. Seventeen patients $(25.8 \%)$ underwent surgical resection followed by HSRS on the tumor bed or adjuvant WBRT, $32(48.5 \%)$ received SRS alone, and $17(25.7 \%)$ WBRT alone. Systemic therapy was performed in $43(65.2 \%)$ cases after local BMs treatment. Characteristic of patients and tumor in relation to the undergone treatment are shown in Table II.

Local control ( $L C)$, distant brain failure (DBF) and overall survival $(O S)$ analysis. The median follow-up time for the whole cohort from BMs diagnoses was 23.4 months (range=0.5-70.1 months) and 32.5 months (range=12.670.11 months) for alive patients. Sixteen $(24.2 \%)$ patients had local recurrence in site of treatment. The median LC time, and the 1, 2, 3 years LC rate were (median not reached $=$ nr), $87.5 \%, 71.2 \%$, and $63.0 \%$ as shown in Figure 1a. The only factor detected as conditioning LC was the breast specific GPA score; the median LC time, the 1, 2, and 3 years LC rate were 28.1 months $(95 \% \mathrm{CI}=2.0-54.2$ months, $81.2 \%, 44.9 \%$, and $0.0 \%$ for GPA $2.5-3, \mathrm{nr}, 91.3 \%, 80.1 \%$, and $72.8 \%$ for GPA score 3.5-4 ( $p$-value=0.04). BDF occurred in $38(42.4 \%)$ patients. The median BDF time, and the 1,2, 3 years BDF rate were 19.7 months $(95 \% \mathrm{CI}=9.0$ - 
Table I. Patients and tumor characteristics.

\begin{tabular}{lcc}
\hline Patients & $\mathrm{N}$. & $\%$ \\
& 66 & 100 \\
\hline Median age years (range years) & $52(31-83)$ & \\
Stage at diagnosis of primary tumor & & \\
$\quad$ I-III & 50 & 75.8 \\
IV & 16 & 24.2 \\
Molecular subtype HER2 & & \\
HR (ER/PR) positive & 27 & 40.9 \\
HR (ER/PR) negative & 39 & 59.1 \\
KPS & & \\
90-100 & 43 & 65.1 \\
70-80 & 22 & 33.3 \\
60 & 1 & 1.6 \\
RPA class & & \\
I & 15 & 22.7 \\
II & 48 & 72.7 \\
III & 3 & 4.6 \\
Breast specific GPA score & & \\
2.5-3 & 28 & 42.4 \\
3.5-4 & 38 & 57.6 \\
Other metastatic site at diagnosis of BMs & 48 & 72.7 \\
Total number of BMs & 130 & 100 \\
BMs treated & & \\
1 lesions & 33 & 50 \\
2 lesions & 14 & 21.2 \\
3 lesions & 7 & 10.6 \\
4 lesions & 12 & 18.2 \\
\hline
\end{tabular}

BMs: Brain metastases; KPS: Karnofsky performance status; RPA recursive partial analyses; GPA: graded prognostic assessment; HR: hormonal receptor.

30.5 months), $29.6 \%, 55.6 \%$. and $70.0 \%$ as shown in Figure 1b. Prognostic factor impacting on BDF was the presence of a single BM compared to more than 1 lesion; the median BDF time, and the 1, 2, 3 years BDF rate were 27.9 months (95\% CI=20.5-35.2 months), $15.5 \%, 41.3 \%$, and $57.3 \%$ for patients with single $\mathrm{BM}$ versus 13.7 months $(95 \% \mathrm{CI}=7.3-$ 20.0 months $), 46.1 \%, 71.8 \%$, and $75.9 \%$ ( $p$-value $=0.04)$. The median OS time, and the 1, 2, 3 years OS were 30.7 months (95\%CI=18.9-42.6 months), 78.5\%, 57.4\%, $43.3 \%$ as shown in Figure 1c. On univariate and multivariate analysis, several factors were identified as influencing survival: KPS, number of BMs, the local treatment performed, the presence of EC metastases at the time of BMs diagnosis, and the administration of trastuzumab more or less combined with a single chemotherapeutic agent. Details about factor conditioning survival are shown in Table III. At the last observation time 24 (36.4\%) patients were alive and $42(63.6 \%)$ had deceased. Among deceased patients, the cause was EC progression in 18 (42.9\%), EC plus brain disease progression in $19(45.2 \%)$, and brain progression only in $5(11.9 \%)$.
Table II. Patients and tumors characteristics in relation to treatment performed.

\begin{tabular}{|c|c|c|c|c|c|c|}
\hline \multirow[t]{2}{*}{ Characteristics } & \multicolumn{2}{|c|}{$\begin{array}{c}\text { Surgery }+ \\
\text { RT } \\
17 \text { pts }\end{array}$} & \multicolumn{2}{|c|}{$\begin{array}{c}\text { SRS/HSRS } \\
\text { only } \\
32 \text { pts }\end{array}$} & \multicolumn{2}{|c|}{$\begin{array}{l}\text { WBRT } \\
\text { only } \\
17 \text { pts }\end{array}$} \\
\hline & $\mathrm{n}$ pts & $\%$ & $\mathrm{n}$ pts & $\%$ & $\mathrm{n}$ pts & $\%$ \\
\hline \multicolumn{7}{|l|}{ Median age years } \\
\hline \multicolumn{7}{|l|}{ KPS } \\
\hline $90-100$ & 13 & 76.5 & 24 & 75 & 6 & 35.3 \\
\hline $70-80$ & 4 & 23.5 & 8 & 25 & 10 & 58.9 \\
\hline 60 & 0 & 0 & 0 & 0 & 1 & 5.8 \\
\hline \multicolumn{7}{|l|}{ RPA } \\
\hline I & 8 & 47.1 & 3 & 9.3 & 4 & 23.5 \\
\hline II & 9 & 52.9 & 28 & 87.5 & 11 & 64.7 \\
\hline III & 0 & 0 & 1 & 3.2 & 2 & 11.8 \\
\hline \multicolumn{7}{|l|}{ Tumor subtypes } \\
\hline ER/PR positive & 6 & 35.3 & 12 & 37.5 & 9 & 52.9 \\
\hline ER/PR negative & 11 & 64.7 & 20 & 62.5 & 8 & 47.1 \\
\hline \multicolumn{7}{|c|}{ Breast specific GPA score } \\
\hline $2.5-3$ & 6 & 35.3 & 14 & 43.7 & 8 & 47.1 \\
\hline $3.5-4$ & 11 & 64.7 & 18 & 56.3 & 9 & 52.9 \\
\hline \multicolumn{7}{|c|}{ Extracranial metastases } \\
\hline Yes & 7 & 41.2 & 26 & 81.3 & 15 & 88.2 \\
\hline No & 10 & 58.8 & 6 & 18.7 & 2 & 11.8 \\
\hline \multicolumn{7}{|l|}{ Number BMs } \\
\hline 1 & 14 & 82.3 & 17 & 53.2 & 2 & 11.8 \\
\hline 2 & 1 & 5.9 & 11 & 34.4 & 2 & 11.8 \\
\hline 3 & 1 & 5.9 & 2 & 6.2 & 4 & 23.5 \\
\hline 4 & 1 & 5.9 & 2 & 6.2 & 9 & 52.9 \\
\hline \multicolumn{7}{|l|}{ Targeted therapies } \\
\hline Yes & 10 & 58.8 & 24 & 75 & 9 & 52.9 \\
\hline No & 7 & 41.2 & 8 & 25 & 8 & 47.1 \\
\hline
\end{tabular}

SRS: Stereotactic radiosurgery; HSRS: hypofractionated stereotactic radiosurgery; WBRT: whole-brain radiotherapy; BMs: brain metastases; KPS: Karnofsky performance status; RPA: recursive partitioning analyses; GPA: graded prognostic assessment; HR: hormonal receptor.

Salvage treatment for intracranial/local progression. Among 16 local brain relapsed patients, 12 underwent further treatments consisting of surgical resection plus HSRS on the tumor bed in 7, WBRT in 3, SRS in 1 and HSRS alone in 1. Of 38 patients with BDF, 14 (36.8\%) received SRS, 14 (36.8\%) WBRT and 10 (26.4\%) did not undergo further local treatment for synchronous EC progression. Systemic therapy was performed in $12(31.6 \%)$ patients.

\section{Discussion}

Breast cancer phenotype strongly influences the outcome of patients with brain metastases, however specific evidence for the management are still lacking (3-6). The published papers are principally population based studies or reviews, regarding all breast cancer subtypes (25-28). The optimal local treatment 

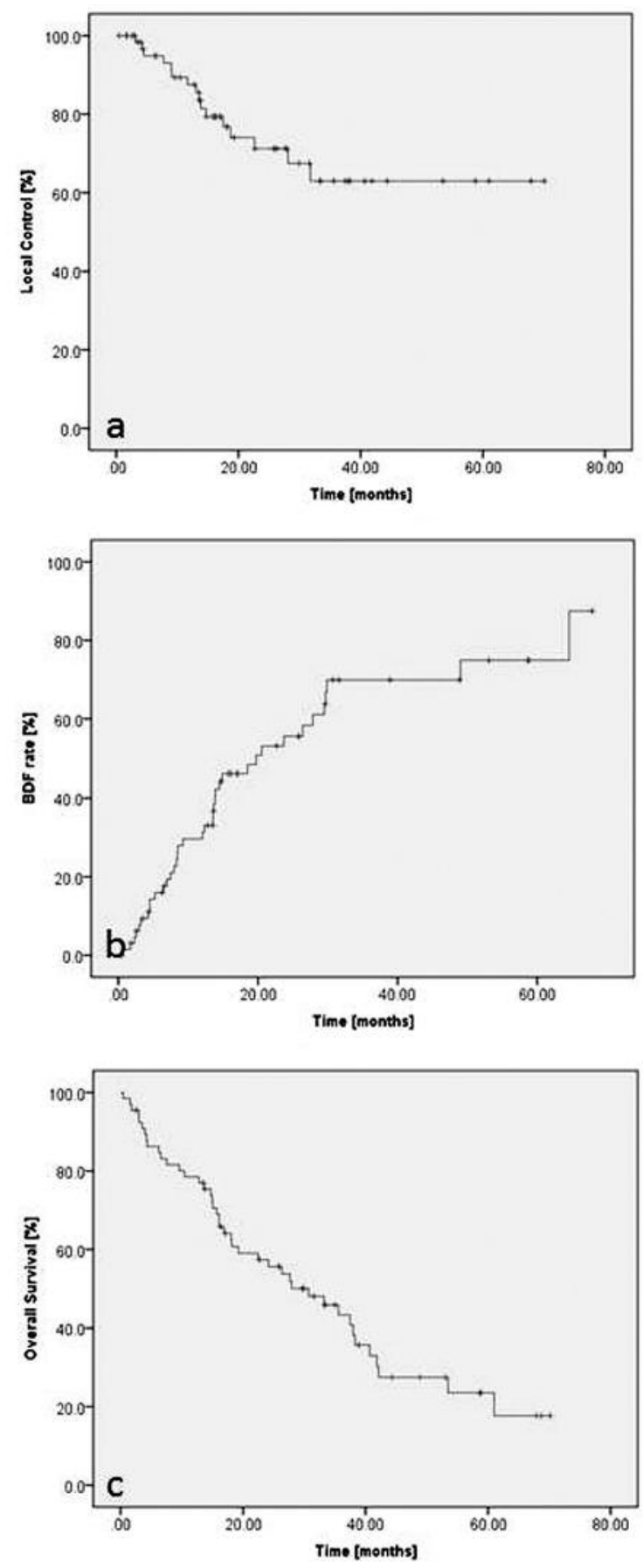

Figure 1. Actuarial analysis: a) Local control $(L C)$, b) Brain distant failure $(B D F), c)$ Overall Survival (OS) for HER2 breast patients with brain metastases (less than 4 ).

and its association with systemic therapy able to influence patient's outcome remains controversial but it is increasingly a challenge for the large number of long-term survivors. Based on these observations we retrospectively analyzed the outcome of HER2BC patients with limited BMs $(\leq 4)$ treated in our institution with surgery and/or RT associated with targeted therapy. Our series appraisal confirmed most recent published data that the greater number of patients $(98.5 \%)$ developed BMs during the course of their disease with a median occurrence time of 40 months from diagnosis of primary HER2BC. In addition, although the large number of patients deceased from EC progression, fatal brain progression only occurred in a discreet number of patients (12\%). However, this knowledge about the HER2BC behavior did not yet allow to define a shared treatment for HER2BC with limited BMs. Several local treatments were used and systemic therapy employed with different results was recorded (15-22). Regarding our cohort, patients with single, large BM, good KPS and controlled EC disease underwent surgical resection followed by WBRT at the first time and HSRS on the tumor bed subsequently, and among these more than half of patients received second line targeted therapy. Conversely, patients with small BMs, poor general condition, and EC progressive disease received SRS or HSRS. Using these criteria comparable results in terms of LC were achieved, confirming the correct treatment choice in relation to clinical and radiological patient's assessment, in the majority of patients. Local control was obtained in about $80 \%$ of patients; relapse in site of treatments occurred in 16 patients at a median time of 12 months from SRS; among these $7 / 16$ underwent surgical resection followed by RT and/or II line targeted therapy, and all were alive at the last follow-up time. These data suggest that in HER2BC patients, with potentially better prognosis, compared to other phenotypes, a more aggressive combined treatment could be considered up front. The survival rate was also highly satisfactory with about $45 \%$ of patients alive at 3 years, favorably compared with previous reports $(26,28)$. As well recognized in several published papers, the KPS and the presence of EC metastases have been recorded as prognostic factors impacting on outcome $(6,8)$. Although there is no literature considering the number of BMs as a factor affecting survival, in our series of patients with a single brain lesion showed a longer OS compared to a number $>1(p=0.05)$. These data could be related with the fact that patients with a single $\mathrm{BM}$ received a most radical treatment, surgery and/or radiosurgery. More relevant, the treatment performed has been demonstrated to significantly impact on survival both on univariate and multivariate analysis. In detail, in patients that had underwent surgery plus RT the median and 3 years OS were 38.3 months and $59.2 \% v s .37 .5$ months and $52.8 \%$ for SRS vs. 14.5 months and $10.6 \%$ for WBRT ( $p=0.002)$; these data could be closely related to the patients selection, more unfavorable for those that received WBRT alone, both for general condition than for brain and EC disease status. Notwithstanding, no consolidate evidences are available regarding the sequential employ of targeted therapy, more than half of our patients received trastuzumab or trastuzumab 
Table III. Factors evaluated as conditioning survival in univariate and multivariate analysis.

\begin{tabular}{|c|c|c|c|}
\hline \multirow[b]{2}{*}{ Factors analyzed } & \multicolumn{3}{|c|}{ Overall Survival (OS) } \\
\hline & Univariate analysis & Multivariate analysis & HR \\
\hline Age & n.s. & n.s. & - \\
\hline Stage at diagnosis & 0.6 & n.s. & - \\
\hline Breast specific GPA score & 0.09 & n.s. & - \\
\hline KPS & $<0.001$ & 0.004 & 2.53 \\
\hline RPA & 0.2 & n.s. & - \\
\hline Hormonal Receptor status & 0.9 & n.s. & - \\
\hline Interval time between $\mathrm{BC}$ diagnosis and $\mathrm{BM}$ occurrence & n.s. & n.s. & - \\
\hline Number of brain metastases & 0.04 & n.s. & - \\
\hline Extra cranial metastases at BM diagnosis & 0.006 & 0.02 & 0.39 \\
\hline Local treatments performed & 0.002 & 0.03 & 0.50 \\
\hline Targeted therapy after local treatments & 0.03 & n.s. & - \\
\hline
\end{tabular}

KPS: Karnofsky performance scale; BC: breast cancer; BM: brain metastases; SRS: stereotactic radiosurgery; HSRS: hypofractionated stereotactic radiosurgery; BMs: brain metastases; RPA: recursive partitioning analyses; GPA: graded prognostic assessment; OS: overall survival; BDP: brain distant progression; LC: local control.

emtansine (TDM1) after BMs treatments. This strategy was found to be effective with $49 \%$ of patients alive at 3 years compared to $33 \%$ of patients that did not receive it $(p=0.03)$. To validate even more the need to keep on systemic therapy even after the local treatments we have to consider the relevance of the presence of EC metastases in affecting outcome. We are aware that our analysis has several and typical limitations related to their retrospective nature in terms of different treatments assessed, the heterogeneity of population, and treatment at recurrence. Nevertheless, considering the lack of literature about the treatment of BMs according to histology or phenotype, and the fair number of patients treated, our study could make a contribution on this topic.

\section{Conclusion}

Patients with limited BMs from primary HER2BC are potentially long-term survivor patients. The control of brain disease is pivotal to allow an optimal control of neurological functions preserving QoL. Our results suggested that the best local strategy seems to be a focalized treatment followed by targeted therapy after local approach. Patients with good KPS, controlled EC disease and single BM could be considered for surgical treatment in first instance. A careful evaluation of prognostic and predictive factors as well as a multidisciplinary evaluation is a valid aid to manage the optimal therapeutic strategy for HER2BC patients with BMs.

\section{Clinical Practice Points}

- The role of local treatments for HER2 breast cancer patients with brain metastases

- 60 patients and 130 brain metastases
- 3-years LC was $63 \%$ and 3-years survival was $43 \%$

- HER2BC patients with good KPS, controlled EC disease and single BM had better outcome.

\section{Conflicts of Interest}

All Authors have no conflicts of interest. No other conflict or source should be disclosed.

\section{References}

1 Crivellari D, Pagani O, Veronesi A, Lombardi D, Nolè F, Thürlimann B, Hess D, Borner M, Bauer J, Martinelli G, Graffeo R, Sessa C and Goldhirsch A; International Breast Cancer Study Group: High incidence of central nervous system involvement in patients with metastatic or locally advanced breast cancer treated with epirubicin and docetaxel. Ann Oncol 12: 353-356, 2001.

2 Graesslin O, Abdulkarim BS, Coutant C, Huguet F, Gabos Z, Hsu L, Marpeau O, Uzan S, Pusztai L, Strom EA, Hortobagyi GN, Rouzier $\mathrm{R}$ and Ibrahim NK: Nomogram to predict subsequent brain metastasis in patients with metastatic breast cancer. J Clin Oncol 28: 2032-2037, 2010.

3 Dawood S, Broglio K, Esteva FJ, Ibrahim NK, Kau SW, Islam R, Aldape KD, Yu TK, Hortobagyi GN and Gonzalez-Angulo AM: Defining prognosis for women with breast cancer and CNS metastases by HER2 status. Ann Oncol 19: 1242-1248, 2008.

4 Niikura N, Hayashi N, Masuda N, Takashima S, Nakamura R, Watanabe K, Kanbayashi C, Ishida M, Hozumi Y, Tsuneizumi M, Kondo N, Naito Y, Honda Y, Matsui A, Fujisawa T, Oshitanai R, Yasojima H, Tokuda Y, Saji S and Iwata H: Treatment outcomes and prognostic factors for patients with brain metastases from breast cancer of each subtype: a multicenter retrospective analysis. Breast Cancer Res Treat 147: 103-112, 2014.

5 Niikura N, Liu J, Hayashi N, Mittendorf EA, Gong Y, Palla SL, Tokuda Y, Gonzalez-Angulo AM, Hortobagyi G and Ueno N: Loss of human epidermal growth factor receptor 2 (HER2) 
expression in metastatic sites of HER2-overexpressing primary breast tumors. J Clin Oncol 30: 593-599, 2012.

6 Sperduto P, Kased N, Roberge D, Chao ST, Shanley R, Luo X, Sneed P, Suh J, Weil R, Jensen A, Brown P, Shih H, Kirkpatrick J, Gaspar L, Fiveash J, Chiang V, Knisely P, Sperduto C, Lin N and Mehta M: The effect of tumour subtype on the time from primary diagnosis to development of brain metastases and survival in patients with breast cancer. J Neurooncol 112: 467472, 2013.

7 Kennecke H, Yerushalmi R, Woods R, Mc Cheang, Voduc D, Speers CH, Nielsen T and Gelmon K: Metastatic behaviour of breast cancer subtypes. J Clin Oncol 28: 3271-3277, 2010.

8 Sperduto P, Kased N, Roberge D, Xu Z, Shanley R, Luo X, Sneed P, Chao S, Weil R, Suh J, Bhatt A, Jensen A, Brown P, Shih H, Kirkpatrick J, Gaspar L, Fiveash J, Chiang V, Knisely J, Sperduto C, Lin N and Mehta M: Effect of tumour subtype on survival and the graded prognostic assessment for patients with breast cancer and brain metastases. Int J Radiat Biol Oncol Phys 82: 2111-2117, 2012.

9 Subbiah IM, Lei X, Weinberg JS, Sulman EP, Chavez-MacGregor M, Tripathy D, Gupta R, Varma A, Chouhan J, Guevara R, Valero V, Gilbert $M$ and Gonzalez-Angulo $A$ : Validation and development of a modified breast graded prognostic assessment as a tool for survival in patients with breast cancer and brain metastases. J Clin Oncol 33: 2239-2245, 2015.

$10 \mathrm{Xu}$ Z, Schlesinger D, Toulmin S, Tyvin R and Sheehan J: Impact of triple-negative phenotype on prognosis with breast cancer brain metastases. Int J Radiat Biol Phys 84: 612-628, 2012.

11 Lin NU, Amiri-Kordestani L, Palmieri D, Liewehr D and Steeg P. CNS metastases in breast cancer: Old challenge, new frontiers. Clin Cancer Res 19: 6404-6418, 2013.

12 Pestalozzi BC, Holmes E, de Azambuja E, Metzger-Filho O, Hogge L, Scullion M, Lang I, Wardley A, Lichinitser M, Sanchez R, Mueller V, Dodwell D, Gelber R, Piccart-Gebhart M and Cameron D: CNS relapses in patients with HER2-positive early breast cancer who have and have not received adjuvant trastuzumab: a retrospective substudy of the HERA trial (BIG 101). Lancet Oncol 14: 244-248, 2013.

13 Vern-Gross TZ, Lawrence JA, Case LD, McMullen KP, Bourland JD, Metheny-Barlow LJ, Ellis T, Tatter S, Shaw E, Urbanic J and Chan M: Breast cancer subtype affects patterns of failure of brain metastases after treatment with stereotactic radiosurgery. J Neurooncol 110: 391-398, 2012.

14. Stemmler HJ, Schmitt M, Willems A, Bernhard H, Harbeck N and Heinemann V: Ratio of trastuzumab levels in serum and cerebrospinal fluid is altered in HER2-positive breast cancer patients with brain metastases and impairment of blood-brain barrier. Anticancer Drugs 18: 23-28, 2007.

15 Pessina F, Navarria P, Cozzi L, Ascolese AM, Maggi G, Riva M, Masci G, D’Agostino G, Finocchiaro G, Santoro A, Bello L and Scorsetti M: Outcome evaluation of oligometastatic patients treated with surgical resection followed by hypofractionated stereotactic radiosurgery (HSRS) on the tumor bed, for single, large brain metastases. PLoSOne 11: e0157869, 2016.

16 Tsao MN, Rades D, Wirth A, Lo SS, Danielson BL, Gaspar LE, Sperduto P, Vogelbaum M, Radawski J, Wang J, Gillin M, Mohideen N, Hahn C and Chang E: Radiotherapeutic and surgical management of newly diagnosed brain metastasis: an American Society for Radiation Oncology evidence-based guidelines. Pract Radiat Oncol 2: 210-225, 2012.
17 Patchell RA, Tibbs PA, Regine WF, Dempsey RJ, Mohiuddin M, Kryscio RJ, Markesbery W, Foon K and Young B: Postoperative radiotherapy in the treatment of single metastases to the brain: A randomized trial. JAMA 280: 1485-1489, 1998.

18 Kocher M, Soffietti R, Abacioglu U, Villà S, Fauchon F, Baumert BG, Fariselli L, Tzuk-Shina T, Kortmann R, Carrie C, Ben Hassel M, Kouri M, Valeinis E, van den Berge D, Collette S, Collette L and Mueller R: Adjuvant whole-brain radiotherapy versus observation after radiosurgery or surgical resection of one to three cerebral metastases: Results of the EORTC 2295226001 study. J Clin Oncol 29: 134-141, 2011.

19 Jagannathan J, Yen CP, Ray DK, Schlesinger D, Oskouian RJ, Pouratian N, Shaffrey M, Larner J and Sheehan J: Gamma Knife radiosurgery to the surgical cavity following resection of brain metastases. J Neurosurg 111: 431-438, 2009.

20 Kelly PJ, Lin YB, Yu AY, Alexander BM, Hacker F, Marcus KJ and Weiss SE. Stereotactic irradiation of the postoperative resection cavity for brain metastasis: A frameless linear accelerator-based case series and review of the technique. Int $J$ Radiat Oncol Biol Phys 82: 95-101, 2012.

21 Eaton BR, LaRiviere MJ, Kim S, Prabhu RS, Patel K, Kandula S, Oyesiku N, Olson J, Curran W, Shu H and Crocker I: Hypofractionated radiosurgery has a better safety profile than single fraction radiosurgery for large resected brain metastases. J Neurooncol 123: 103-111, 2015.

22 Ling DC, Vargo JA, Wegner RE, Flickinger JC, Burton SA, Engh J, Amankulor N, Quinn A, Ozhasoglu C and Heron D: Postoperative stereotactic radiosurgery to the resection cavity for large brain metastases: clinical outcomes, predictors of intracranial failure, and implications for optimal patient selection. Neurosurgery 76: 150-157, 2015.

23 Shaw E, Scott C, Souhami L, Dinapoli R, Kline R, Loeffler J and Farnan N: Single dose radiosurgical treatment of recurrent previously irradiated primary brain tumors and brain metastases: Final report of RTOG protocol 90-05. Int J Radiat Oncol Biol Phys 47: 291-298, 2000.

24 Mohammadi AM, Schroeder JL, Angelov L, Chao ST, Murphy ES, Yu JS, Neyman G, Jia X, Suh J, Barnett G and Vogelbaum $\mathrm{M}$ : Impact of the radiosurgery prescription dose on the local control of small ( $2 \mathrm{~cm}$ or smaller) brain metastases. J Neurosurg 126: 735-743, 2017.

25 Phillips C, Jeffree R and Khasraw M: Management of breast cancer brain metastases: A practical review. The breast 31: 90-98, 2017.

26 Miller JA, Kotecha R, Ahluwalia MS, Mohammadi AM, Chao ST, Barnett GH, Murphy E, Vogelbaum M, Angelov L, Peerboom D and Suh J: Overall survival and the response to radiotherapy among molecular subtypes of breast cancer brain metastases treated with targeted therapies cancer 123: 2283-2293, 2017.

27 Martin AM, Cagney DN, Catalano PJ, Warren LE, Bellon JR, Punglia RS, Claus E, Lee E, Wen P, Haas-Kogan D, Alexander B, Lin $\mathrm{N}$ and Aizer A: Brain metastases in newly diagnosed breast cancer a population-based study JAMA Oncol 3: 1069-1077, 2017.

28 Geraud A, Xu HP, Beuzeboc P and Kirova YM: Preliminary experience of the concurrent use of radiosurgery and T-DM1 for brain metastases in HER2-positive metastatic breast cancer. J Neurooncol 131: 69-72, 2017.

Received September 1, 2017 Revised September 22, 2017 Accepted September 25, 2017 